\title{
A Debye function based powder diffraction data analysis method
}

\section{Giannini1 ${ }^{1, *}$, A. Cervellino², A. Guagliardi, F. Gozzo ${ }^{4}$, D. Zanchet ${ }^{5}$, T. Rocha ${ }^{6}$ and M. Ladisa ${ }^{7}$}

\author{
${ }^{1}$ Istituto di Cristallografia, IC - CNR, via Amendola 126/O Bari, Italy \\ ${ }^{2}$ Laboratory for Neutron Scattering, ETH Zurich and Paul Scherrer Institut, CH-5232 Villi- \\ gen PSI, Switzerland \\ ${ }^{3}$ Istituto di Cristallografia, IC - CNR, via Amendola 126/O Bari, Italy \\ ${ }^{4}$ Swiss Light Source Paul Scherrer Institut CH-5232 Villigen PSI, Switzerland \\ ${ }^{5}$ Laboratório National de Luz Sincrotron LNLS Caixa Postal 6192 - CEP 13084-971, Cam- \\ pinas, SP - Brazil. \\ ${ }^{6}$ Laboratório National de Luz Sincrotron LNLS Caixa Postal 6192 - CEP 13084-971, Cam- \\ pinas, SP - Brazil and IFGW-UNICAMP, Campinas-SP, Brazil \\ ${ }^{7}$ Istituto di Cristallografia, IC - CNR, via Amendola 126/O Bari, Italy \\ * Contact author; e-mail: cinzia.giannini@ic.cnr.it
}

Keywords: powder diffraction, nanocrystals, Debye function

\begin{abstract}
The ability of tailoring the dimensional regime of nanoparticle ensembles represents a landmark achievement in nanoscience. A key aspect is, however, the development of appropriate methods of data analyses of these complex objects. We present, here, a new method for interpreting powder diffraction data, based on the use of the Debye Function and suitable to treat data collected on virtually any nanocrystalline material. The method has been presently limited to single-domain particles. Applications of technological interest are also discussed.
\end{abstract}

\section{Introduction}

Powder diffraction data analysis techniques based on the use of the Pair Distribution Function or the Debye Function (DF) are total scattering techniques. Their independence of the structure periodicity has made these techniques a suitable tool for structural studies of disordered (up to amorphous) materials. Only recently these techniques have been applied to increasingly complex materials, such as quasicrystals ${ }^{1-2}$ and nanocrystals $(\mathrm{NCs})^{3-5}$.

We here present a DF based powder diffraction method, suitable to interpret data collected with different scattering radiation (X-ray, neutrons, electrons, light). The basics of the method are given elsewhere ${ }^{4-5}$ and here applied to the structural study of specific examples:

- CdSe single-size ultra stable mass-selected nanoparticles (magic number clusters) used as building blocks in functional nano materials ${ }^{6-7}$;

- magnetic iron oxide tetrapods ${ }^{8}$, important in nanomedicine applications 
- Au nanocrystals (NCs) capped with alkanethiols of different lengths (hexa-C6, dodecane$\mathrm{C} 12$ and hexadecane-C16) are presented as a particular case of microstructural quantitative analysis application.

\section{The DF based pattern calculation}

The diffraction pattern is obtained by summing up three components: the structural $I^{c}(q)$, the amorphous $I^{a}(q)$ and the background $I^{b}(q)\left(I^{\text {total }}(q)=I^{c}(q)+I^{a}(q)+I^{b}(q)\right.$, where $\left.q=2 \sin \theta / \lambda\right)$. The structural pattern $I^{c}$, calculated using the Debye formula, is the sum of the pattern intensity diffracted by all the phases indexed by $X$ in the samples: $I^{c}(q)=\sum_{X} X^{c}{ }_{X}(q) S_{X}, S_{X}$ representing the scale factor. Furthermore, a real sample is correctly described in terms of a size distribution. Consequently, the intensity calculation has to be extended to $n$ clusters of increasing size: $I^{c}(q)=\sum_{X} \sum_{n} I_{X ; n}^{c}(q) L_{X}(n)$ where the sum over the cluster sizes is weighted, in this model, by a lognormal size distribution function $L_{X}(n)$. The calculation of $I_{X ; n}^{c}(q)$, described in details in ref. 4-5, requires, for each cluster, the knowledge of all the interatomic distances. Distances databases have been calculated and stored for some crystallographic prototypes (diamond, rocksalt, fcc, rutile, zincblende and wurtzite ${ }^{9}$ ) as well as for specific non-crystallographic ones ${ }^{4-5}$. For each material having one of the listed prototypes, it is possible to use the same corresponding database, re-scaling the interatomic distances with the correct unit cell and substituting the proper atomic species. Sampling of the interatomic distances has been studied and optimized in terms of computing time in order to make this approach suitable for whole profile pattern fitting procedures ${ }^{5}$.

The background term $-I^{b}(q)$ - is a linear combination of low-order Chebyshev polynomials. The amorphous term $-I^{a}(q)$ - represents the residual particle scattering, coming from any possible small disordered (or differently ordered) subdomain of the real nanoparticles, such as surface defects and occasional ad-atoms ${ }^{11}$.

\section{Application}

\section{CdSe magic number clusters}

Magic number clusters are single-sized, stable nanoparticles, with well-defined structures that can be identified with atomic precision and whose physical and chemical properties strongly depend on their particular atomic arrangements. X-ray powder diffraction spectra of CdSe size-selected clusters were collected the Swiss Light Source Materials Science (SLSMS) beamline powder diffraction (PD) station. Samples were 1-1.5 nm nanoparticles, prepared with a novel synthesis route in a particular mixture of surfactants and much lower temperatures ${ }^{7}$ with respect to conventional syntheses. At this size extreme, the ratio of surface-to-volume atoms exceeds 50\%, adding important surface stress contributions, which can strongly affect the internal structure of these clusters. In addition to this, elemental analysis had revealed that our samples were richer in $\mathrm{Cd}$. A possible serie of zinc-blende like cluster models have been produced, calculating the corresponding distance databases. These models have been refined against the experimental data in order to select the best one and to estimate the surface contributions (see figure 1). 


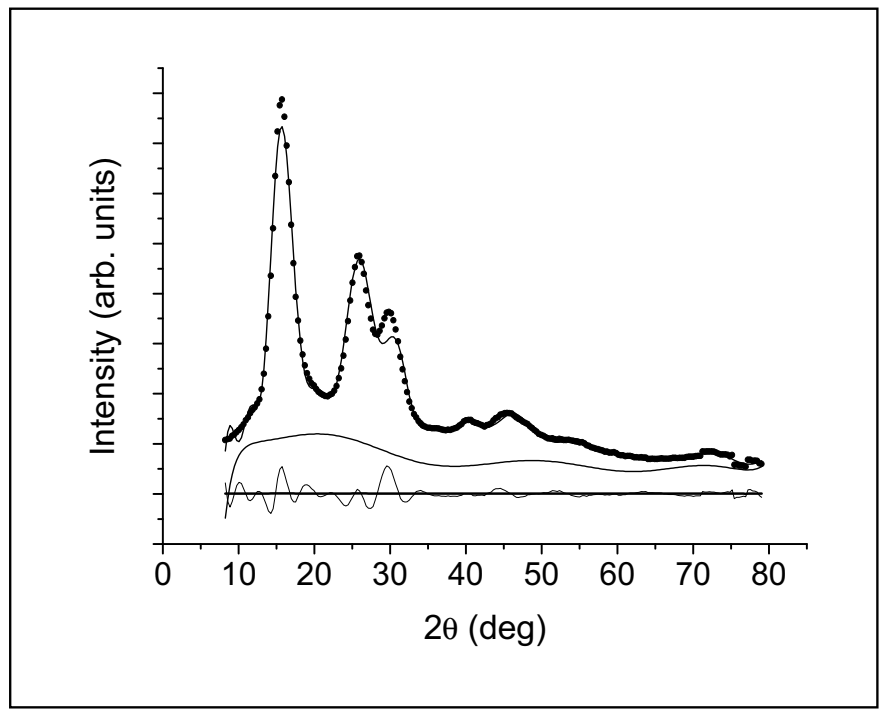

Figure 1. $1.4 \mathrm{~nm}$ - CdSe diffraction pattern. Experimental data(crosses) and refined pattern (red line).

\section{Iron Oxide Tetrapods}

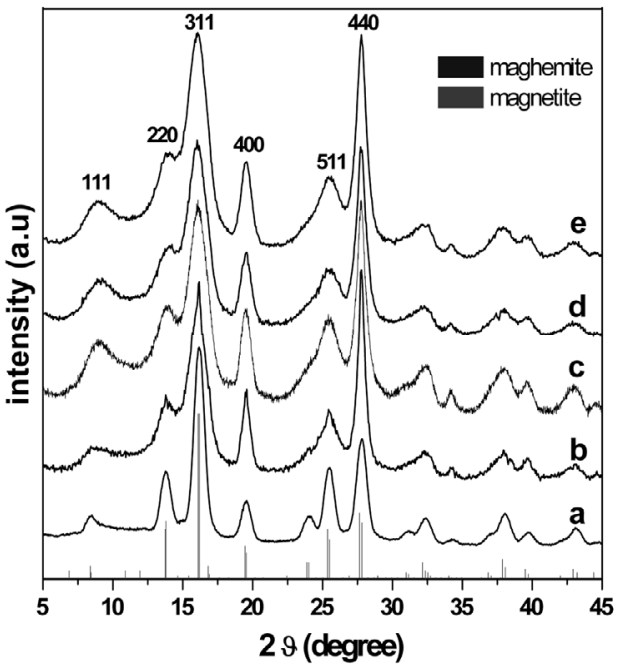

Monocrystalline maghemite nanocrystal tetrapods were synthesized, systematically tuning width and length of the tetrapod arms via reaction parameters. Tetrapods (TPs) were found to grow preferentially along the [111] axis directions, as shown by transmission electron microscopy. Powder diffraction data were collected at the SLS-MS beamline PD station (see figure 2).

TPs exhibit substantial alteration in the relative peak intensity $\left(I_{h k l}\right)$ and widths of several reflections, in comparison with the sphere (a). A gradual increase in the $\mathrm{I}_{220}: \mathrm{I}_{511}$ and especially in the $\mathrm{I}_{440}: \mathrm{I}_{311}$ ratio accompanies the transition from the spherical to the TP shape with progressively longer

Figure 2. Experimental data for TPs of increasing shape anisotropy (from $b$ to $e$, data collected on spherical clusters (a) are included for comparison). 
branches. It can also be noted that the (111) and (220) peak maxima slightly shift to higher angles.

Two families of tetrapod-shaped finite-size nanocrystal structure atomistic models were generated, with arms oriented along the [111] and [110] directions. The respective powder diffraction patterns were computed (see figure 3 ) and compared with the data.
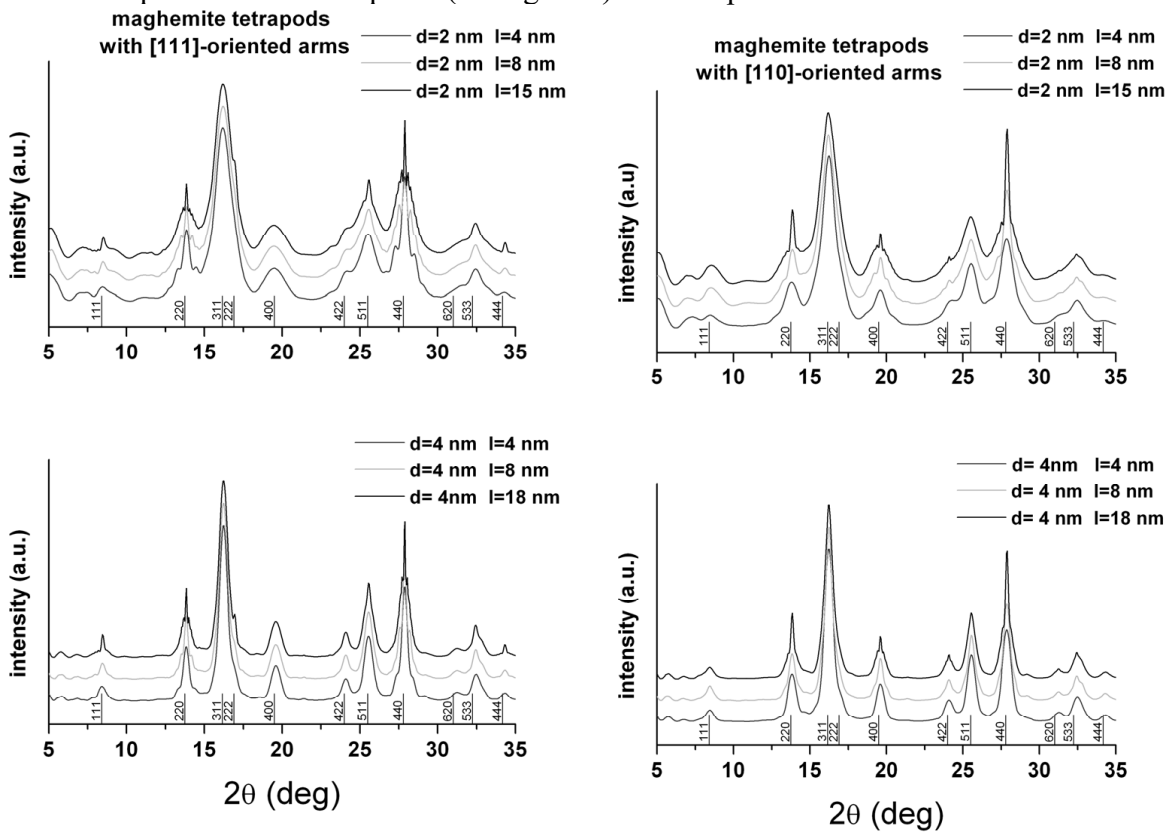

Figure 3. Calculated XRPD patterns of iron oxides tetrapods.

For the [111]-oriented tetrapods, the longer the arm, the sharper the (111), (222) and (444) peaks. Similarly, for the [110]-oriented tetrapods, the (220) and (440) peaks narrow. However, other reflection peak widths, such as the (400) and (422) for both orientations and the (220) and (440) for the (111)-orientation, are affected by the domain elongation. Simulated XRD patterns suggest that TPs with [111]-oriented arms could coexist with TPs with [110]elongated branches. We stress the fact that XRPD was the only technique capable of highlighting this possible sample feature.

\section{Au nanocrystals}

$1.5 \mathrm{~nm} \mathrm{Au} \mathrm{NCs} \mathrm{capped} \mathrm{with} \mathrm{hexa-C6,} \mathrm{dodecane-C12} \mathrm{and} \mathrm{hexadecane-C16} \mathrm{thiols} \mathrm{have} \mathrm{been}$ studied with XRPD collecting the data at the LNLS synchrotron source in Campinas. In order to extract information about the influence of the capping layer on the size, strain and structure distributions, synchrotron powder data were analyzed together with Extended X Ray Absorption Fine Structure (EXAFS) and Small Angle X Ray Scattering (SAXS).

SAXS data were analysed by using the GNOM software assuming polydisperse spherical particles. EXAFS data analysis was performed by using the ATHENAS/ARTHEMIS pack- 
age. The XRD data were analysed by our approach, where the domain size distribution, strain-size dependence and structure type concentrations were refined together with background coefficients and thermal isotropic factors. Four model structures were considered: cuboctahedron (CUBO), icosahedron (ICO), decahedron (DECA) and blunt decahedron (BDEC). The BDEC structure was obtained from DECA by removing the equatorial belt of atoms. An amorphous contribution was also included to describe a potential atomic surface reconstruction and/or superficial strain fields due to the different molecules. SAXS and EXAFS data analysis suggested that samples have the same size and size distribution and a similar $1 \% \mathrm{Au}-\mathrm{Au} \mathrm{NND}$ contraction, with respect to the bulk value, independently on the surfactant molecule length. XRPD analysis confirmed these results and in addition pointed out important differences among samples related to structural concentrations (see figures 4-5 for one of the investigated samples).

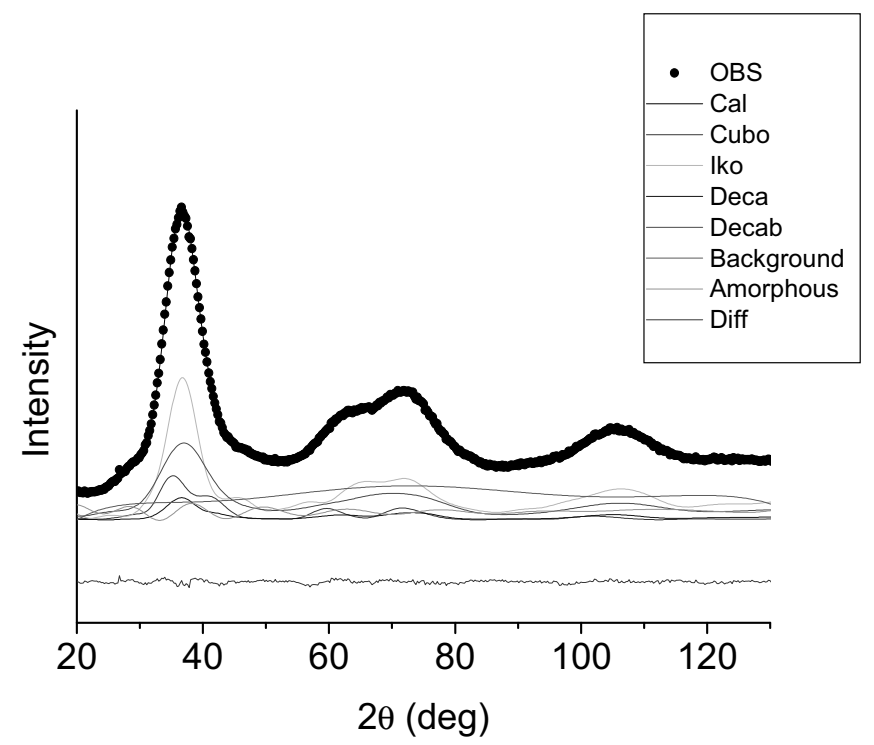

Figure 4. Experimental and calculated XRPD patterns of AuC6B (6x hexanethiol) nanoparticles. GoF $=1.7$

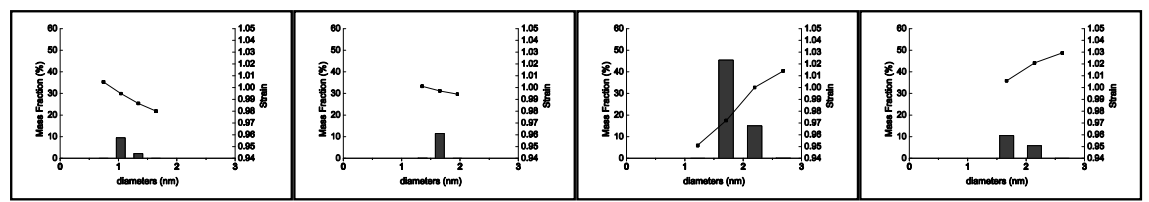

Figure 5. Size and strain distribution for sample AuC6B (6x hexanethiol) for the four structures: CUBO (16.39\%), ICO (60.37\%), DECA (11.55\%) and BDEC (11.68\%). 
While the major population in the Au-C6 is the icosahedron, for Au-C12 sample is the truncated decahedron and for the $\mathrm{AuC} 16$ one is the cuboctahedron. These results suggest that the cap length is likely to affect the particle atomic arrangement.

\section{Concluding remarks}

Crystallographic methodologies are evolving to treat diffraction data collected on nanomaterials. The approach here proposed offers a new tool for the microstructural characterization of nanoparticles in terms of size, strain, shape and structure. The approach has been developed to deal with any nanomaterials and any size. Applications to several practical cases of technological interest are in due course, which will allow us to tune and improve the actual potentialities of the approach on the base of realistic demands.

\section{References}

1. Proffen, Th., Billinge S. J. L., Egami T. and Louca D., 2003, Z. Kristallogr., 218, 132.

2. Brühne S., Uhrig E., Luther H.A., Assmus W., Brunelli M., Masadeh A.S., Billinge S.J.L., 2005, Zeitschrift für Kristallographie, 220, 962.

3. Zanchet D., Hall B.D., Ugarte D., X-Ray Characterization of nanoparticles, WileyVCH, Ed. Zhong Lin Wang, pp. 13-35.

4. Cervellino A., Giannini C., Guagliardi A., 2003, J. Appl. Cryst., 36,1148.

5. Cervellino A., Giannini C., Guagliardi A., 2006, J. Comp. Chem., 27, 998.

6. Kasuya A., Sivamohan R., Barnakov Y.A., Dmitruk I.M., Nirasawa T., Romanyuk V. R., Kumar V., Mamykin S.V., Tohji K., Jeyadevan B., Shinoda K., Kudo T., Terasaki O., Liu Z., Belosludov R.V., Sundararajan V. and Kawazoe Y, 2004, Nature Materials, 3, 99.

7. M. Zanella, S. Kudera, C. Giannini, A. Rizzo, Y. Li, G. Gigli, R. Cingolani, G. Ciccarella, W. J. Parak and L. Manna, 2006, Advanced Materials (in press).

8. Cozzoli P. D., Snoeck E., Garcia M. A., Giannini C., Guagliardi A., Cervellino A., Gozzo F., Hernando A., Achterhold K., Parak F. G., Cingolani R., and Manna L., 2006, Nanoletters, 6, 1966

9. http://cst-www.nrl.navy.mil/lattice/prototype.html

10. Zanchet D., Hall B.D., Ugarte D. (2000) J. Phys. Chem., B 104, 11013.

11. Cervellino A., Giannini C., Guagliardi A., Zanchet D. 2004, Eur. Phys. B, 41, 485 